\title{
Chemotherapy in the treatment, control, and elimination of human onchocerciasis
}

\author{
This article was published in the following Dove Press journal: \\ Research and Reports in Tropical Medicine \\ 21 October 2014 \\ Number of times this article has been viewed
}

\author{
Tarig B Higazi' \\ Timothy G Geary² \\ Charles D Mackenzie 3,4 \\ 'Department of Biological Sciences, \\ Ohio University Zanesville, Zanesville, \\ $\mathrm{OH}$, USA; ${ }^{2}$ Institute of Parasitology, \\ McGill University, Montreal, QC. \\ Canada; ${ }^{3}$ Center for Neglected \\ Tropical Diseases, Liverpool School \\ of Tropical Medicine, Liverpool, UK; \\ ${ }^{4}$ Department of Pathobiology \& \\ Diagnostic Investigation, Michigan \\ State University, East Lansing, MI, USA
}

Correspondence: Tarig B Higazi Department of Biological Sciences, Arts and Sciences, Ohio University, I425 Newark Rd, Zanesville,

$\mathrm{OH} 4370 \mathrm{I}$, USA

$\mathrm{Tel}+\mathrm{I} 740588$ I533

Fax + I $740453616 \mid$

Email higazi@ohio.edu
Abstract: Onchocerciasis treatment is one of the most positive stories in tropical medicine although major challenges remain to reaching the ultimate goal of disease elimination. Such challenges are to be expected when the therapeutic goal is to kill and safely remove a large multistage, efficient, metazoan infectious agent such as Onchocerca volvulus that has an exceptionally complicated relationship with its host. Successful control of onchocerciasis has often been hampered by host reactions following chemotherapy, that can sometimes cause significant tissue pathology. Presence of other filariae, particularly Loa loa, in endemic onchocerciasis-treatment areas also poses severe problems due to adverse reactions caused by drug-induced death of the coincident microfilariae of this usually clinically benign species. Although ivermectin has been very successful, there is a need to enhance the progress toward elimination of onchocerciasis; new drugs and their efficient use are keys to this. The permanent absence of Onchocerca microfilaridermia, defined as the lack of resurgence of skin microfilarial loads after treatment, is the ultimate characteristic of a useful new chemotherapeutic agent. Several drugs are under investigation to achieve this, including the reassessment of currently available and previously tested agents, such as the antibiotic, doxycycline, which targets the adult parasites through its anti-Wolbachia endosymbiont activity. Flubendazole, a benzimidazole derivative approved for treatment of human gastrointestinal nematodes, is also being considered for repurposing as a macrofilaricide to aid in the achievement of eradication. The managerial challenges existing at the population level also need to be addressed; these include drug-distribution fatigue, the need to include noncompliant people, civil unrest in endemic areas, political cross-border issues, restrictions of age and pregnancy, and complications due to integration with other treatment programs. It is likely that a panel of chemotherapeutic options, new and old, supported by strong and effective distribution systems will be the best way to address challenges of treatment and elimination of this infection. Future research should also address management of treatment and control, and consider how new treatment paradigms can be incorporated to meet time lines set for global elimination by 2025 .

Keywords: mass drug administration, ivermectin, macrofilaricides, challenges

\section{Introduction}

Onchocerciasis, caused by infection with the filarial nematode Onchocerca volvulus (Figure 1), has been one of the most recognized tropical nematode diseases, largely through the emotive images of the blind patients in onchocerciasis-endemic areas. It is also a prominent example of successful transmission control achieved through the important, now common, mass drug administration (MDA) approach to distributing drugs to endemic populations; these community-wide drug distribution programs have been in place since 1987 . This approach has drastically reduced the prevalence 


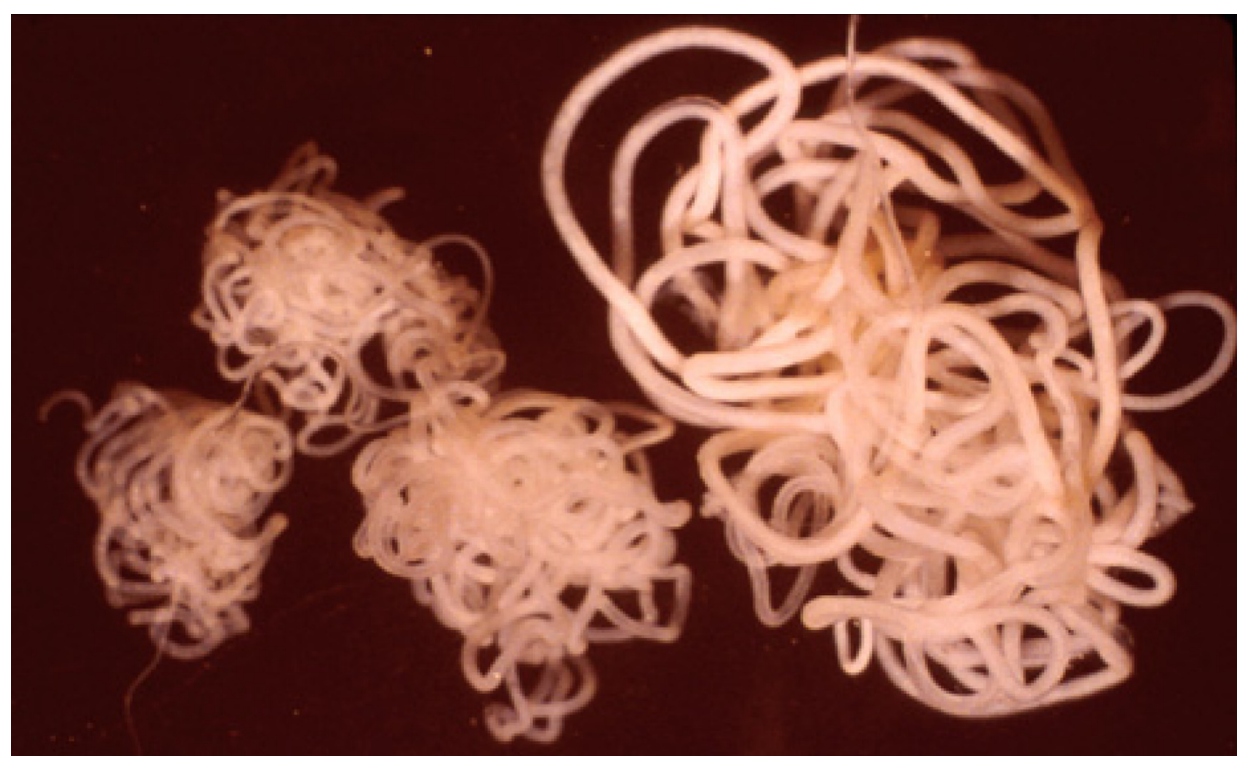

Figure I Onchocerca volvulus filarial parasites (three adult males and one adult female).

of overt disease, brought infection to the point of elimination of its transmission, and begun to break the infection cycle permanently in many areas. The use of the MDA approach with ivermectin, for the first time with onchocerciasis, has changed the face of tropical medicine and the provision of health care services to populations that have been the most under-served in the world. A concerted global effort is now underway to finally eliminate onchocerciasis, along with other parasitic diseases such as lymphatic filariasis, from their respective endemic areas. Elimination, ie, breaking of transmission for 3 years at unsustainably low levels, ${ }^{1}$ has now occurred in Colombia ${ }^{2}$ and Ecuador ${ }^{3}$ as well as in specific endemic foci in Africa. ${ }^{4}$ Other countries are likely to reach the state of complete breaking of transmission, especially in Latin America where, for example, Mexico is poised to join the successes of Colombia and Ecuador. ${ }^{5,6}$ Indeed, this is a most exciting time in tropical medicine, when such possibilities as elimination are even considered as achievable goals. This review summarizes the path taken to this point with regard to the chemotherapeutic treatment of river blindness, and suggests some ways that it may change as we approach the goal of elimination of onchocerciasis as a public health concern by $2025 .{ }^{7}$

Onchocerciasis poses an exceptional challenge to therapy in part because of its complex life cycle. It is a vector-borne disease, transmitted to humans by the bite of infected black flies in the genus Simulium; this biology means that there is a reservoir of infected vectors outside of humans, which can be the cause of the transmission of this parasite moving to new areas. Adult worms live in anatomically discrete tissue nodules in humans, some of which are palpable. Adults may live for more than 15 years, but contribute little to pathology. Mated females release many thousands of larval stages over their life span, termed microfilariae (mf), that primarily reside in the skin. Host inflammatory and immunological reactions associated with the destruction of $\mathrm{mf}$ stage in the skin and eye are responsible for significant pathological changes in these tissues. Treatment should optimally remove both adult and $\mathrm{mf}$ stages from the host without exacerbating the pathology associated with parasite death and degeneration, which is severe when there are large numbers of acutely dying parasites. Should such drugs be available, prospects for the eradication of onchocerciasis are enhanced by the fact that $O$. volvulus only infects humans; the absence of an animal reservoir means that such chemotherapeutic interventions need to be directed only at humans, with black fly control as a matter of additional support.

Several drugs have been used over the past 70 years to treat patients infected with $O$. volvulus. Many of them are independently toxic to the host at the same dose levels used to cure the infection, while others have been plagued by serious adverse reactions associated with the death of the parasite. Finding a safe drug requires a good understanding of the mechanisms by which the host reacts to the death of the parasite, including both the adult and the $\mathrm{mf}$ stages. Sadly, it can be argued that much of the exacerbation of eye disease in Africa related to onchocerciasis resulted from the extensive use of diethylcarbamazine (DEC), which was for many years the drug of choice for this disease. As discussed below, the reactions associated with DEC are particularly 
severe and tissue damaging. Therefore, the discovery and development of improved agents for onchocerciasis must carefully consider treatment-induced pathology and any potential for inducing serious adverse clinical reactions.

Several recent reviews ${ }^{8-10}$ describe approaches to finding new drugs for filariasis, including onchocerciasis. We focus here on the current status of chemotherapy and the fundamental challenges to developing new treatments, discussing the biological and logistic problems presented by this complicated tropical infection. Improving treatment of this parasitic disease must be considered in the context of the renewed global efforts to eliminate many such parasitic infections under the banner of neglected tropical diseases (NTDs). However, it could be argued that onchocerciasis is far from being "neglected" as there have been major attempts to control it since 1974.

\section{Challenges of treating onchocerciasis}

Onchocerciasis is a challenge to treat for many reasons (Table 1), including the ability of the worm to potentially resist treatment, its adaptability in terms of biochemistry, and the many consequences of breaking the extraordinary biological "agreement" that exists between the worm and its host that can result in the pathology that is the basis of this disease. In addition, there are the extraordinary challenges in distributing a drug or a procedure to people living in some of the most remote areas of the tropics. As onchocerciasis control has largely been based on insecticide and anthelmintic deployment, the monetary aspects of supplying and using insecticides and drugs in such large numbers require large donations of material and financial support as well as the development of managerial and logistic capacities in difficult places in hot climates (Table 1). Like many tropical diseases, onchocerciasis patients typically lack financial resources to pay for treatment, and the provision and distribution of therapeutic interventions is an endeavor that falls to national services and not one that enters the realm of commerce.

It must also be noted that there is a distinct difference between chemotherapy for an intestinal parasite, one that is essentially lying outside the body, and that of one residing within host tissues, such as $O$. volvulus. This distinction is evident when addressing the many issues regarding the development and administration of a chemotherapeutic agent that need to be resolved, such as safety, bioavailability, and route and frequency of administration, for a treatment against a tissue-based parasite compared with a gut-based organism.
Table I Key factors in the chemotherapy of onchocerciasis

\begin{tabular}{ll}
\hline Item & $\begin{array}{l}\text { Key factors in the chemotherapy of } \\
\text { onchocerciasis }\end{array}$ \\
\hline I & Reactions to dying microfilariae are responsible for \\
the pathology: these occur in untreated infections and \\
are naturally and are often exacerbated after therapy- \\
induced parasite degeneration \\
The ultimate goal for treatment of onchocerciasis is a \\
sustained suppression of dermal microfilariae; this may \\
come through an agent directed at the adult worms that \\
can survive in natural infections for long periods of time \\
A range of drugs for different epidemiological situations \\
would greatly enhance a program's ability to reach \\
elimination in as short a time as possible \\
IV $\quad \begin{array}{l}\text { Optimal management of drug distribution and treatment } \\
\text { coverage is a vital component for achieving success } \\
\text { The approaches to medical management of individual } \\
\text { onchocerciasis patients may differ considerably for the } \\
\text { approaches to treating endemic communities, although } \\
\text { this distinction is likely to narrow as programs approach } \\
\text { elimination } \\
\text { The current global NTD approach changes in both } \\
\text { positive and negative ways the progress toward the } \\
\text { treatment and elimination of onchocerciasis } \\
\text { Loaiasis is a special challenge with regards ivermectin } \\
\text { treatment and the handling of this infection needs to be } \\
\text { carefully addressed by program management teams }\end{array}$ \\
VI
\end{tabular}

Abbreviation: NTD, neglected tropical disease.

Clearly, the treatment parameters for intestinal parasites can differ dramatically from those for tissue-based parasites.

\section{Biological challenges}

Nematodes adapted to living inside humans have a complex biology. As a group, filariae are extraordinarily well adapted, and most often show little outward evidence that they are present in the host. Some, such as Loa loa, can be present for many years without major clinical signs. This is not to say that there are no subclinical effects present in apparently unaffected hosts; indeed, loaiasis causes a number of detectable changes, although the overall clinical effect is minor. Even with lymphatic filariasis, where a small proportion of the infected people show the dramatic clinical presentations of lymphedema and hydrocele, it is likely that all the infected people actually have some degree of pathological change at the tissue level, albeit occult. It is as though host and parasite in these "quiescent" infections have come to a mutual agreement for an almost peaceful coexistence. Thus, most filarial infections cause relatively little acute disease but instead can have serious, debilitating long-term consequences. From this perspective, one could argue that $O$. volvulus is in fact not as well adapted to its host as are many other filariae in that it has a clearly observed clinical presentation in virtually all 
infected people. The intolerable itching and skin destruction along with, in some cases, ocular tissue damage reflect its relatively poor adaption to its human host.

Central to this biological adaption that underscores the host-parasite interface is the phenomenon of manipulation of the host's immune system by the parasite. Immune responses to onchocerciasis, including suppression and activation, have been described. The lack of an animal model has been a hindrance to research, and much remains to be learned about this interface. Medical science has not yet been able to adequately manipulate the host immune system to develop immunotherapies, such as vaccines, for prevention of this infection.

\section{Pathological challenges}

The major clinical lesions in human onchocerciasis result from tissue reactions associated with the death of the $\mathrm{mf}$ present in the skin and eye, where dying and degenerating $\mathrm{mf}$ induce inflammatory responses in these tissues. In the natural untreated infection, these damaging events happen slowly and continually with the natural turnover of $\mathrm{mf}$ numbers $-\mathrm{mf}$ have a life span of 6-12 months and are constantly replenished by fertile females. The local inflammation resulting from the death of mf over time can gradually cause permanent damage in the tissues. Unfortunately, these tissue-destroying inflammatory events occur in host tissues that are particularly sensitive and often nonregenerative, such as the retina, and thus lead to clinically evident and permanent damage, including blindness and dermal degeneration.

When the death of mf occurs "en masse" due to treatment with microfilaricidal agents, the clinical response can be extremely severe. This is why early chemotherapeutic approaches for onchocerciasis were plagued with adverse events, some of which were extremely severe and even fatal. ${ }^{11}$ Thus, a successful treatment for this disease should destroy the parasites in a manner that does not elicit severe host responses, a challenge that contributes greatly to the difficulties of developing new antifilarial drugs (Table 1). This fundamental problem plagued drug treatment for river blindness until the adoption of ivermectin (see below). DEC caused severe reactions associated with the rapid death and destruction of mf. This effect was particularly problematic because DEC also has an independent effect on the host inflammatory response in a way that is incompletely understood but is thought to involve the arachidonic acid pathway and is associated with anaphylactoid conditions. ${ }^{12}$

An additional complication is that onchocerciasis patients can be coinfected with other filarial parasites, which can themselves induce problems during chemotherapy. The most serious coinfections with onchocerciasis occur with L. loa, which can reach very high levels of $\mathrm{mf}$ in blood (eg, 100,000 mf/mL). This problem is most commonly seen at a community level in MDA programs where the area under treatment in question is co-endemic for L. loa. Treatment of patients with $L$. loa present at this high level often induces adverse clinical responses; severe reactions, including death, can occur in these hyper-microfilariaemic patients after treatment with ivermectin. ${ }^{11}$ It is thought that the significant mass of dying mf in vital tissues such as the brain may be related to the pathogenesis in this syndrome. More than 250 people have died from a severe encephalopathy that followed within 48 hours of treatment with ivermectin; ${ }^{13}$ such reactions were also seen in the past when DEC was used. Obviously, a primary challenge to finding a safe chemotherapeutic approach for onchocerciasis, at least with Loa coinfected patients (or those living in co-endemic regions), is to find agents that kill adults but not $\mathrm{mf}$ and thus avoid mf-dependent severe adverse events. Alternatively, one could develop methods to identify and avoid treating those people who have high loads of circulating $\mathrm{mf}$, if a microfilaricidal agent is to be deployed (Table 1).

\section{Logistic challenges}

The global approach to the treatment of onchocerciasis has evolved to rely on annual or twice-yearly treatment with ivermectin in MDA campaigns. The original purpose for the donation of ivermectin by Merck \& Co, Inc. (Whitehouse Station, NJ, USA) was to limit transmission and reduce pathology (especially for onchocercal blindness) in highly endemic areas. This program has enjoyed remarkable success, leading to a new phase aimed at controlling dermatological disease, and then more recently the adoption of the goal of elimination of this infection from all endemic areas. ${ }^{7}$ Whereas this is being achieved in Latin America, many challenges remain to achieving this goal in Africa and Yemen. Aside from the obvious need for a safe and effective drug approach to treatment, there are logistic challenges of distributing such a drug to everybody in an endemic area effectively enough to break transmission and to eventually eliminate the parasite (Table 1). Dominant among the obvious logistic challenges is the difficulty of delivering drugs to difficult geographic areas, often amidst civil disturbances, and ensuring that noncompliant people are included in the treatment program and thus prevent the retention of 'hidden' reservoirs of infection. Compliance and extent of coverage are central issues for the achievement 
of the goal of elimination. ${ }^{15}$ Strong government support and very effective management systems are required to overcome these challenges; maintaining this support in the face of declining case rates to achieve the ultimate goal is likely to be a significant challenge to eventual disease eradication.

The logistic challenges in onchocerciasis therapy differ between two situations: that of treating individual infected patients on the one hand and the contrasting case of providing treatments without diagnosis through MDA in endemic areas. A good example of how good management can overcome the challenges presented by MDA is evident in the onchocerciasis programs in Latin America. In Ecuador, the endemic area was in a difficult jungle riverine location with very active transmission through a voracious vector. Nevertheless, MDA with ivermectin twice a year eliminated transmission; ${ }^{3}$ this program developed a very close relationship with the endemic villages and managed to maintain a very high level of coverage over 17 years. In contrast, the endemic focus in the Amazonian jungle, where it is difficult to maintain access and contact with the nomadic population of American Indians (Yanomamo), is proving difficult to eliminate. ${ }^{16}$ Different approaches to treatment and the associated logistical support will most likely be needed for this challenging population.

Additional logistic issues come into play when the disease exits across the borders of two or more endemic countries; examples of this are the Amazonian jungle focus in South America mentioned above, and also in the endemic areas of Eastern Sudan and Western Ethiopia in North Africa. Comprehensive epidemiological studies are needed to verify whether such foci are isolated from each other or indeed constitute a single continuous disease focus; whether the vectors active in these sites are the same across these borders; and what the characteristics are of the respective vector breeding sites and their closeness to communities. Cross-border foci will require a high degree of political, managerial, and scientific coordination between the respective parties involved to ensure complete success in eliminating onchocerciasis at these sites.

A special situation arises when treating onchocerciasis patients who are not living in endemic countries; here there is a challenge in obtaining the appropriate drug (currently ivermectin), which is not readily available in non-endemic countries, and usually needs to be obtained through official (government) sources. With the increase in global travel, including to onchocerciasis-endemic countries, physicians in non-endemic countries must be aware of the possible need to diagnose and treat these infections; this is not entirely the case today.

As mentioned above, significant political and financial components are needed for treatment of this disease at the field level. In addition, there has been a recent expansion of global organizations devoted to eliminating and controlling many of the major NTDs. Although the MDA program for onchocerciasis was the first to contribute to the well-being of people living in the tropics, other NTDs have been joined to these early efforts. The lymphatic filariasis program, which is a large elimination program involving 160 million people, ${ }^{17}$ is perhaps the closest to the onchocerciasis-elimination programs, and indeed shares the current chemotherapeutic approach, using ivermectin (plus albendazole) in countries that also have onchocerciasis present; DEC is used in place of ivermectin in countries that are onchocerciasis-free. Integration of all these new MDA programs presents major logistical challenges for in-country medical institutions that require continuing strong political and financial support to resolve.

\section{History of onchocerciasis treatment}

When developing new approaches to treatment, one should be fully aware of what has been used in the past (Figure 2). Understanding why previous approaches failed might allow us to improve these agents and perhaps repurpose them using modern technological approaches; this may be a more rapid approach to drug development compared to de novo discovery and development of completely new agents. The history of onchocerciasis treatment until the time of ivermectin was plagued with adverse host reactions largely due to reactions associated with the death of $\mathrm{mf}$; however, suramin, an agent endorsed as the "standard treatment" in highly endemic areas such as Sudan in the 1950s and 1960s, caused additional and direct side effects that could be lethal. ${ }^{18}$

An original, and still used, approach to treatment of onchocerciasis was to remove the adult worms that live in palpable nodules situated under the skin. This useful intervention was used by early investigators, ${ }^{19}$ although it is only partially effective as there are likely to be additional nonpalpable nodules present deeper in the body. Surgical campaigns to remove nodules were very common in Central America after the discovery of the disease there. These campaigns were, however, very unpopular with patients. Removing nodules surgically may be more effective in cases of recent infections, such as those in non-endemic individuals, as there is likely to be a single nodule present. Long-term residents of endemic areas are likely to have many more nodules than 


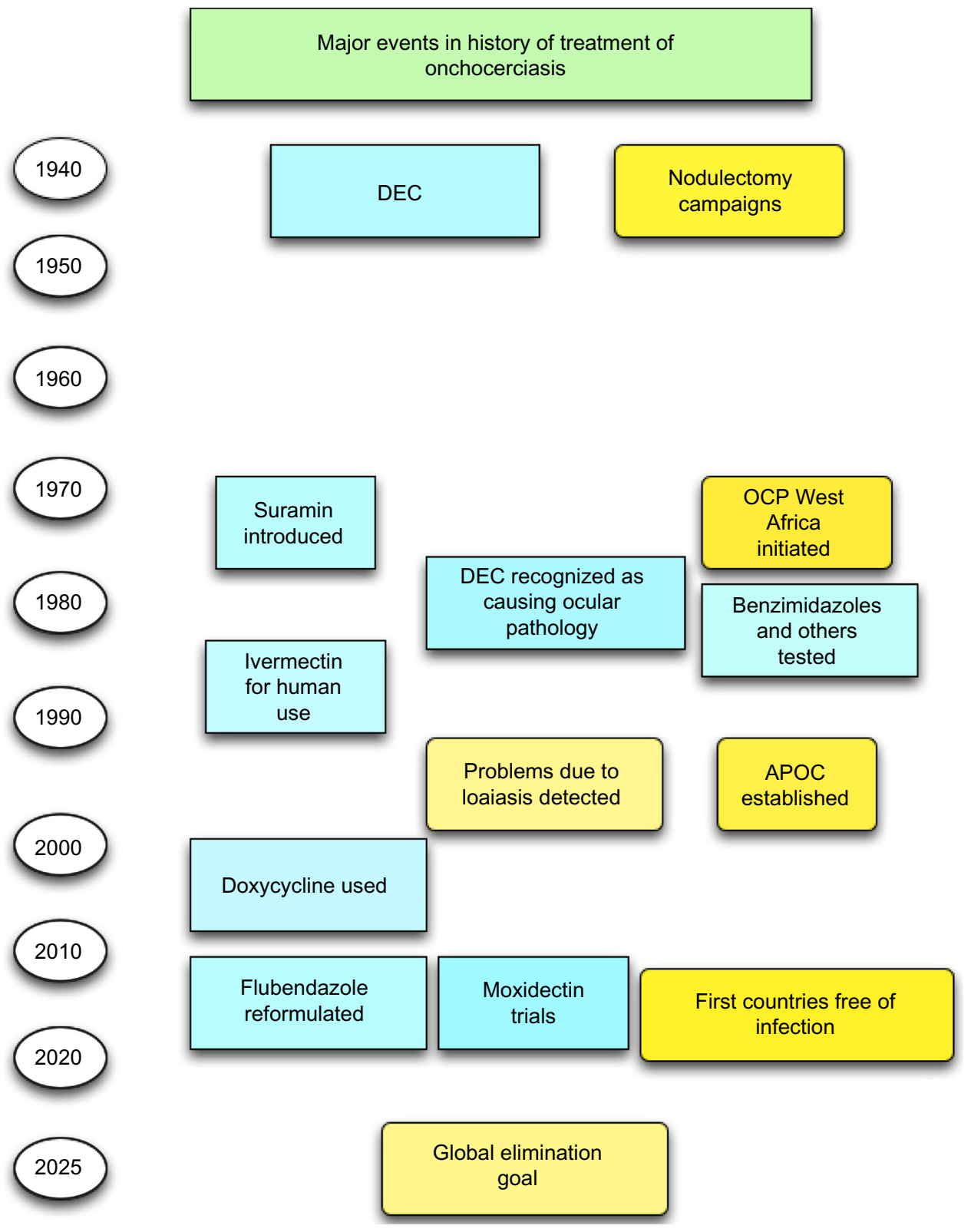

Figure 2 History of onchocerciasis treatment and control.

Note: Events associated with specific drugs are shown in blue, and more general events shown in yellow.

Abbreviations: APOC, African Program for the Control of Onchocerciasis; DEC, diethylcarbamazine; OCP, Onchocerciasis Control Program.

recently infected individuals such as western travelers and expatriates. An example at the field level of the ineffectiveness of surgical campaigns comes from the Ecuadorian focus, where in the 1980s the disease was extremely severe and increasing rapidly in endemicity despite strong and active nodulectomy campaigns. ${ }^{20}$ In this case, the distribution of ivermectin over a period of 17 years was required to eliminate transmission of $O$. volvulus. ${ }^{3}$ Nevertheless, the removal of nodules is justified as a "standard treatment" procedure as long as it can be done using medically appropriate procedures. Nodules have been known to cause pressure pain when impinging on bone points or other sensitive places; they are also disfiguring and aesthetically displeasing. It is not uncommon for infected people to attempt self-removal of nodules by inserting objects such as nails through the skin into the swellings; this totally inappropriate treatment should be actively discouraged.

Chemotherapy came into play for the treatment of onchocerciasis with the work of Luis Mazzotti in pioneering studies in Central America, where he used DEC. ${ }^{21} \mathrm{He}$ was also the first to describe the characteristic adverse dermal reactions that occur in patients after treatment with this drug. These reactions include pruritus, papular eruptions, and hyperemia of the skin, and are associated with the death 
of $\mathrm{mf}$ in the skin. These anaphylactoid-type reactions have become known as "Mazzotti reactions"22 and are seen with other microfilaricidal drugs, including ivermectin, although the manifestations with this drug are typically mild and tolerable. ${ }^{23}$ These dermal reactions were also the basis for a "provocative test": a low dose of DEC is given to elicit these pruritic papular reactions in patients infected with O. volvulus. It is crucial to note that mild dermal responses seen in some ivermectin-treated people are clinically and pathogenically distinct from reactions occurring in cases of hyper-microfilariaemic loaiasis.

The arsenical Mel W was suggested for onchocerciasis in the early 1960 s by Janssens ${ }^{24}$ and Friedheim, ${ }^{25}$ but was found to be unacceptable for reasons of toxicity and only modest efficacy. The polyanionic compound suramin is another comparatively toxic agent; although its action in filariasis is still unclear, it was used as a macrofilaricide until the 1970 s for the treatment of onchocerciasis. This was commonly used in Sudan and was known as the "Sudan regimen"; the drug was given intravenously once a week for 6 weeks. ${ }^{26}$ Little is known of the true efficacy of this inherently toxic agent, which was very unpopular with both patients and medical staff. Nevertheless, research was carried out on this drug, as it was the only macrofilaricide available. ${ }^{27,28}$ However, toxicity issues associated with suramin finally stopped its use and it is no longer approved for this indication.

The next significant step related to onchocerciasis and DEC came from studies carried out in South Sudan in the early 1980s. Led by ophthalmologist Alan Bird, the team showed that patients who had $\mathrm{mf}$ present in ocular tissues experienced severe vascular changes and loss of vision after treatment with the standard dose of DEC. ${ }^{29}$ This finding led to the restriction of DEC use in countries where onchocerciasis is present. To escape this unacceptable adverse event, work continued in the early 1980s on new DEC formulations that would not induce severe ocular changes. One approach was based on a report from Liberia suggesting that DEC applied as a topical cream was a safe and effective approach that avoided adverse reactions. ${ }^{30}$ However, this material caused devastating dermal and systemic responses when tried in patients in South Sudan; topical DEC may in fact induce more severe local Mazzotti reactions than oral dosing, and is certainly not a suitable treatment. ${ }^{31}$ A more positive consequence of this topical approach was the development of the current DEC patch test ${ }^{32}$ as a diagnostic method..$^{33,34}$

A number of drugs approved for treatment of gastrointestinal nematodes were tested in the early 1980s, including benzimidazoles (Figure 2). Supported by the World Health
Organization (WHO) through their "MacroFIL Programme" tasked with finding a macrofilaricide, human studies were carried out in Mexico to evaluate mebendazole, flubendazole, and levamisole in comparison to DEC. ${ }^{35,36}$ Albendazole was studied separately in Venezuela. ${ }^{37}$ The most promising of this chemical class tested here was flubendazole. This drug has very low oral bioavailability, necessitating intramuscular injection as the route of delivery in the original Mexican study. The formulation caused unacceptable injection-site reactions in some of the patients and the drug was not advanced to development even though it was highly efficacious against adult $O$. volvulus. ${ }^{38}$ This early observation led to a significant investment in synthesis of flubendazole prodrugs, which could provide improved oral bioavailability to deliver adequate systemic exposure to flubendazole. None of these compounds was advanced to human testing, although some were efficacious in animal models. ${ }^{38}$ Other candidate macrofilaricides were tested in the 1980s in human trials, including compounds from formerly Ciba-Geigy, now Novartis International AG (Basel, Switzerland) $)^{39}$ and antiparasitic drugs such as chloroquine and the organophosphate metrifonate. ${ }^{40,41}$ None were sufficiently safe or effective to warrant further development.

The introduction of the very successful veterinary drug ivermectin for onchocerciasis control in 1987 (Figure 1) led to deprioritization of macrofilaricide discovery programs. ${ }^{42}$ Like DEC, ivermectin is a highly effective microfilaricide (Figure 2), and crucially, it caused only mild Mazzotti reactions. It also did not induce the damaging ocular changes associated with DEC, perhaps because mf killing by ivermectin only begins a few days after dosing and requires an active immune system, a system usually considered to be less active in the ocular tissues. It is also believed that few $\mathrm{mf}$ actually die in the eye, but that they move into dermal tissues where they are more actively affected by this drug. Ivermectin is thought to act by interrupting the secretory apparatus of $\mathrm{mf}$, blocking the release of protective molecules that keep the host immune system at bay. ${ }^{43,44}$ It also is known to prevent the release of late stages of developing $\mathrm{mf}$ from the reproductive tract of female worms, ${ }^{45}$ although the mechanism underlying this effect, which lasts for many months, remains ill-defined. A single ivermectin dose of $160 \mu \mathrm{g} / \mathrm{kg}$ brings dermal mf loads in most people to very low levels for at least 6 months, although it should be noted that this response is quite heterogeneous, ranging from minimal changes to very effective reduction; various factors are at play here, including host variation. $\mathrm{mf}$ generally begin to repopulate the skin 7-9 months after dosing, although they do not reach pretreatment levels. A study in Sudan showed that the immune system 
is likely to be important in the effectiveness of ivermectin in reducing dermal parasite loads. ${ }^{46}$

One major recent contribution to onchocerciasis chemotherapy has been the focus on destroying the endosymbiont Wolbachia that these parasites need for survival. Although Wolbachia had been identified in O. volvulus in the $1970 \mathrm{~s},{ }^{47}$ it was not until Hoerauf et $\mathrm{al}^{48-50}$ used doxycycline to generate a macrofilaricidal effect that these bacteria became a target for treatment (Figure 2). In addition, it has been suggested that the gold-containing compound, auranofin, a drug approved over 25 years ago for use in humans, may be a useful macrofilaricidal agent; humans trials in onchocerciasis with this drug are expected in the near future.

\section{Currently used anthelmintics}

Two drugs are used routinely for MDA in onchocerciasisendemic areas: ivermectin and albendazole - this is a WHO-approved combination for treating lymphatic filariasis patients in Africa, some of whom also have onchocerciasis. Although we have a fairly good understanding of the effects of ivermectin on $O$. volvulus, especially on the mf stage, little is known about the effects of albendazole on $O$. volvulus, and there is little evidence that it has clinical utility in onchocerciasis in the currently approved regimen. A study in Venezuela with albendazole in onchocerciasis showed a partial effect, ${ }^{37}$ supportive studies are lacking.

Ivermectin is a semisynthetic derivative of an avermectin, which are fermentation products of Streptomyces avermitilis. It is produced as $3 \mathrm{mg}$ and $6 \mathrm{mg}$ tablets for use in onchocerciasis (labeled as Mectizan; Merck \& Co, Inc.), for lymphatic filariasis when combined with albendazole, and for strongyloidiasis (labeled as Stromectol; Merck \& Co, Inc.). For onchocerciasis, it is given at a dose of $120-200 \mu \mathrm{g} / \mathrm{kg}$. It is metabolized by the liver and its metabolites are almost exclusively excreted in the feces, with a half-life of around 18 hours; peak plasma values are observed at around 4 hours after dosing. Higher doses do not appear to be more effective, ${ }^{51}$ although more frequent dosing than the standard approach of once a year used in Africa does appear to produce higher efficacy, and dosing every 3 months improved clinical conditions compared to annual treatment. Ivermectin remains contraindicated for children under 5 years of age (although it has been used extensively in very young animals in veterinary medicine), for pregnant women, and the infirm; in addition, it should not be used in individuals carrying high loads of L. loa mf. ${ }^{13,14}$ Dermal reactions, ie, mildly pruritic papular responses, do occur after ivermectin treatment in many infected patients, but these reactions take many hours longer to develop than those occurring with a classical DEC-induced Mazzotti reaction, and are very much milder in intensity. Given the fact that MDA is carried out in field environments, it is important to realize that pregnant women may actually inadvertently receive the drug; retrospective studies have shown that this has not resulted to date in any adverse outcomes in the pregnancy or the developing infant. ${ }^{52-54}$

Other than ivermectin, the only other available filaricidal agent with documented efficacy in onchocerciasis is the antibiotic doxycycline, which is administered as a daily dose of 100-200 mg for 4-6 weeks. ${ }^{50,55}$ As it is a tetracycline, doxycycline use is age-restricted; combined with the additional challenge of the need for a long duration of therapy, doxycycline in its current form is unlikely to alone achieve elimination. Doxycycline eliminates the prokaryotic endosymbiont Wolbachia that is present in most filarial nematodes, including $O$. volvulus (but not L. loa), and is required for survival (Figure 2). Doxycycline has been shown to be a useful adjunct approach in recent studies in Africa. ${ }^{50}$ Research is ongoing to find new antibiotics or antibiotic combinations that could reduce the duration of therapy required for doxycycline to a regimen more compatible with field use. Although it requires a long course of treatment and cannot be given to individuals younger than 9 years of age or to pregnant or lactating women, doxycycline is nonetheless the only macrofilaricide available for human use.

\section{Managing adverse reactions}

Chemotherapy of onchocerciasis has been plagued by adverse reactions, and it is important to consider how to manage them. Reactions with ivermectin are minimal and rarely require further medical attention. The dermal pruritus sometimes associated with ivermectin treatment is usually reduced satisfactorily with antihistamines.

In contrast, the severe adverse events associated with treatment of patients with hyper-loaiasis (Table 1) remain mechanistically mysterious, and little is known about the most-appropriate therapy for affected patients. ${ }^{56}$ Many patients who suffer these sequelae die or suffer irreversibly central nervous system consequences. ${ }^{14}$ Some are reported to have suffered from secondary infections and have succumbed some weeks after the initial treatment. Certainly, the most important action is immediate hospitalization and provision of essential medical support. Questions remain about how these cases might be treated to prevent the severe clinical sequelae. Limited studies in experimental models suggest that corticosteroids and aspirin, commonly given to these 
patients, are not particularly useful in this condition. It is possible that these patients are immunocompromised, which may have contributed to the high L. loa mf loads. However, optimal nursing care and antibiotic use should be always used to minimize bedsores or other infections.

\section{Components of a successful treatment program}

When considering the characteristics needed for successful treatment of onchocerciasis, one must distinguish treating an individual under full medical support compared with treating populations at the village level where there is only minimal medical support. Obviously, many components overlap between these two target groups, but there are also essential differences. Treatment must be safe and effective and fit all the requirements of the medical world today (Table 1). It must be remembered that useful adjunct approaches can help manage this complex disease, such as vector control in village transmission sites, and the use of ameliorating agents to reduce pruritus and improve skin condition. However, antiparasite chemotherapy clearly remains the central approach for onchocerciasis treatment.

It is perhaps obvious to say that the optimal form of a new treatment is an oral tablet that could be given to children as well as adults, including pregnant women, in a single dose (Table 2); this might be too high a bar to set if the goal is to quickly develop a new drug that could contribute to eradication within the desired time frame. Other characteristics, such as stability at high temperatures, low cost-of-goods, and efficacy against only adult stages of the parasite could be included in an optimal target product profile. However, it is important to consider what is available now and what might be a useful improvement on the current situation. Certainly high on the list of characteristics is a drug that would safely and with a minimal number of treatments kill adult Onchocerca. There is a concern that quickly and simultaneously killing multiple adult filariae would induce unacceptable tissue reactions such as abscesses. This has been seen with lymphatic filariasis, in which treatment with an adulticidal regimen of DEC in Haiti induced lymphangitis and abscesses. ${ }^{57}$

The minimal efficacy required of a macrofilaricide is also a matter of discussion. ${ }^{9,10}$ Obviously, $100 \%$ killing is optimal, but this may very well be an unrealistic goal. Currently, we have no drug that kills adult worms safely in only a few doses (one to three). In this context, a macrofilaricide with $90 \%$ efficacy in a limited-dose regimen would be an extraordinary and most-welcome improvement for national programs and for the treatment of infected individuals (Table 2). Data are not available that would allow us to set a lower limit on the degree of macrofilaricidal efficacy that would be of value to the elimination of onchocerciasis; modeling may be informative and should be pursued.

It is important to stress that ivermectin markedly reduces the $\mathrm{mf}$ load and causes little to no pathology. This has led to drastic reductions in symptoms and transmission, an amazing improvement from the time when only DEC was available. However, it would be a considerable achievement to be able to reduce the time that programs need to distribute ivermectin from the current 12 or more years to perhaps two to three rounds of treatment with a macrofilaricide. It should be noted that doxycycline, as noted, is a macrofilaricide; there is no doubt that it kills adult worms, but the fact that it needs to be distributed on a daily basis for 4-6 weeks must be regarded as less than optimal.

Table 2 Characteristics of a chemotherapeutic agent that would enhance the current onchocerciasis-elimination global programs

\begin{tabular}{|c|c|c|}
\hline & Optimal characteristics & Minimum (acceptable) characteristics \\
\hline Route of administration & Oral dosing & Intramuscular dosing \\
\hline Schedule & Single dose & a) 2-5 daily doses or b) I dose monthly for 3 months \\
\hline Efficacy & Prevents microfiladermia in all patients & $\begin{array}{l}\text { Prevents microfiladermia in all patients for more than } \\
\text { I } 5 \text { months }\end{array}$ \\
\hline Outcome measure & $\begin{array}{l}\text { Complete suppression of dermal microfilaria that } \\
\text { is sustained for over } 2 \text { years }\end{array}$ & $\begin{array}{l}\text { Complete suppression of microfilaridermia for } \\
\text { I } 5 \text { months }\end{array}$ \\
\hline Target population & All people above 2 years of age & All males above 9 years of age \\
\hline General characteristics & $\begin{array}{l}\text { Complying with the safety requirements in the } \\
\text { countries to be used }\end{array}$ & $\begin{array}{l}\text { Complying with the safety requirements in the countries } \\
\text { to be used }\end{array}$ \\
\hline Formulation & $\begin{array}{l}\text { Stable tablet with more than } 4 \text { years longevity at } \\
\text { temperatures and humidities experienced in endemic } \\
\text { countries }\end{array}$ & A cold chain formulation \\
\hline Safety & Usable in all patients, including those with loaiasis & $\begin{array}{l}\text { Not usable in pregnancy nor in those under the age of } \\
9 \text { years }\end{array}$ \\
\hline Programmatic use & Mass drug administration & Individual patient treatment \\
\hline
\end{tabular}

Note: These characteristics are given as an indication of the type of drug that would be of assistance to global programs directed at elimination. 
It is also important to discuss the possibility of the development of resistance to ivermectin. This unfortunate phenomenon has been commonly recorded in the veterinary literature, ${ }^{42}$ and is probably the result from the extensive use of this anthelmintic in cattle, sheep, and also in dogs. ${ }^{58}$ There have been reports of "atypical" or low responders (or resistance) in Ghana ${ }^{59}$ and possibly in Cameroon ${ }^{60}$ in relation to its use in onchocerciasis; however, proof that this phenotype is due to genetic or true resistance has not as yet been clear. These "poor responders" may reflect genetic drift rather than resistance within parasite populations; more work is clearly indicated to determine the status of resistance to ivermectin in $O$. volvulus.

A discussion of a new drug for the treatment of onchocerciasis in MDA programs must include a consideration of its ease of production as well as a funding source for its production and distribution to calculate the cost effectiveness of its use. These parameters may change over time. For example, when praziquantel was first introduced for the treatment of schistosomiasis, the cost of the drug was initially high. However, as it became incorporated into field programs, improvements in logistics and production reduced the cost of the drug to a practical level. This is likely to happen with any new drug for NTDs, and must be factored in cost-effectiveness analysis.

An important aspect of onchocerciasis treatment that nevertheless is rarely discussed is for the alleviation of the intolerable itching and dermal changes that occur in most patients; this is particularly true for patients who suffer from the particularly severe form of the disease known as "sowda". ${ }^{61-63}$ Unfortunately, most patients who are infected with $O$. volvulus live in areas where palliative treatments (such as antihistamines, corticosteroids, and dermal creams) are not readily available. Certainly, patients with the "sowda" form require considerable dermatological treatment and attention; these patients often injure themselves whilst trying to suppress the severe itching and commonly have breakages to the skin. Similarly, the cracks and folds that appear in affected skin require antifungal and antibiotic ointments.

The fact that onchocerciasis can be challenging to diagnose may be an issue in individual patient management, especially with individuals seen outside endemic zones. The attending clinician may not be completely sure of a positive diagnosis in these cases. A drug that can be safely given to people who are suspected rather than defined as having onchocerciasis would be an advantage.

So what kind of chemotherapeutic agent is needed? Although there is general agreement that a macrofilaricide is needed, the therapeutic end point is not necessarily the death of adult parasites but instead is the permanent removal of $\mathrm{mf}$ from the skin $-\mathrm{mf}$ are the causative stage of pathology and are responsible for transmission. In this context, a drug that permanently sterilized adult females would be as useful as a drug that killed them. The best indication of success in elimination is the lack of $\mathrm{mf}$ in the skin rather than the lack of nodules, of infective larvae, or of particular antigens or circulating antibodies. Although these latter entities are useful indicators, the most important outcome for onchocerciasis is absence of mf. A possible target product profile for such a chemotherapeutic agent to achieve elimination of onchocerciasis is shown in Table 2. In addition, adequate safety and stability goals must also be reached for use of a drug in a developing country environment.

Field programs have become very used to a once a year regimen with a very safe drug for treatment and control of filarial diseases such as onchocerciasis. Indeed, the treatment of other tropical diseases such as tuberculosis, HIV, leprosy, etc is far more complicated, and we should thus be open to more complex approaches to onchocerciasis if they could lead to a rapid eradication. There is also an unfortunate tendency to underestimate the capabilities of village health staff in developing countries to administer complex treatment plans; whereas complex treatment approaches are obviously quite challenging in areas undergoing civil disturbance or conflict and in remote rural areas, in stable countries the capabilities of local medical facilities are remarkably robust, and more complicated regimens than once a year can be easily accommodated.

In 2002, it was believed by the scientific community that the tools existed, based on chemotherapy, that could eliminate onchocerciasis from Latin America but this could not yet be achieved in Africa. This belief for the Latin American situation is becoming true and many lessons can be learnt from the New World disease that could possibly assist the programs in Africa. Perhaps the fundamental characteristic of success in the American situation has been consistent, well managed high coverage with the drug (ivermectin) in more than one cycle per year. The showcase example of this is success in the Ecuadorian focus ${ }^{3}$ where twice-a-year treatment with consistently high coverage (usually $>85 \%$ ) eliminates the transmission in an endemic area with a very voracious vector (Simulium exiguum) that is thought to be similar to the situation regarding transmission in much of Africa with Simulium damnosum. The inclusion of additional rounds of treatment with ivermectin per year, such as has been achieved with four rounds per year in Mexico, ${ }^{6}$ also 
appears a very useful strategy that could be incorporated in many places in Africa. Although, as described in detail elsewhere in this review, there is a concerted effort to find new chemotherapeutic agents - especially a macrofilaricide, perhaps better described as an agent that can maintain the suppression of the production of microfilariae - it should be remembered that efficient use of ivermectin, as applied in the Americas, can be successful in eliminating transmission, although admittedly this usually requires a long period of time, a factor that is becoming less acceptable in the current health policy climate of "quick successes".

\section{Successes}

The use of ivermectin in onchocerciasis control programs is a tremendous success story in tropical medicine. Although there is a need for a drug targeted at adult worms to achieve elimination goals, the stellar record of these programs is an undeniable and remarkable tribute to medical science, social science, and particularly to the many thousands of people who have contributed to the actual distribution of ivermectin in the field: the community distributors. These programs have set the standard for similar approaches to other NTDs. The discovery and distribution of ivermectin have been arguably among the most important events in tropical medicine in the last half-century and laid the basis for a needed change in the way medicines are distributed in developing countries. The effects of these programs extend well beyond the reduction in blindness and dermatitis; they have promoted the development of health care systems for those living in rural areas of developing, and often war torn, countries. It is sobering but important to remember that a number of people, particularly distributors in the field, have lost their lives in this laudable cause.

The first indicator of the major successes in the treatment of onchocerciasis was the recognition 15 years ago that no children were suffering from ocular disease due to this infection in treated areas of West Africa. The past decade saw the success of national programs in Latin America, where treatment of the relatively small foci of onchocerciasis showed that ivermectin given twice a year could eliminate the parasite as long as high coverage was achieved ( $>80 \%)$. Both Colombia and Ecuador ${ }^{3}$ are now in all likelihood free of the disease. In Africa, some endemic foci, such as the Nubian Desert in Sudan ${ }^{4}$ and parts of West Africa, ${ }^{64}$ are now free of transmission of onchocerciasis following the dedicated and intensive distribution of ivermectin.

Paralleling these successes, there has been a gradual change in the goals of onchocerciasis control programs. These programs began in 1987 to reduce the level of blindness, then in the mid-1990s changed to reducing the prevalence of onchodermatitis. In 2002, it was proposed that onchocerciasis could be eliminated from Latin America, but the elimination of the disease and transmission in Africa was not yet possible. By 2010, global opinion had again changed and a concerted effort to eliminate the infection from Africa was endorsed. ${ }^{7}$ However, it is still recognized that challenges remain in achieving the goal of elimination of onchocerciasis by 2025 . As noted, these challenges include adverse reactions to coinfections with loaiasis, funding issues, and the challenges of long-term sustainability and program fatigue.

\section{Changes to current approaches}

Two areas warrant consideration in a discussion of how current approaches to onchocerciasis treatment might be enhanced to achieve the goal of elimination by 2025 . Firstly, current national programs should be supported and assisted in any way they need to ensure high drug coverage and program efficiency; this is of course vital in countries that have special difficulties, such as ongoing civil disturbance, very large or difficult-to-reach populations needing treatment in remote areas, or comparatively undeveloped health systems.

Secondly, the provision of a panel of drugs and procedures that can be adjusted to fit the needs of particular endemic areas would be a major advance. Rather than a single therapy approach, which has been the strategy for the past 25 years involving ivermectin, more diverse approaches that better respond to local needs may be needed. It is generally accepted that a monotherapeutic approach to treatment will probably not suffice to reach elimination, and that other drugs, especially a new macrofilaricide, would be a very welcome addition. Tailoring therapies and other supportive activities, such as vector control, to the particular needs of each endemic area in a local context will be of most use, particularly for addressing control in hypoendemic areas, which have usually been ignored in MDA programs. A panel of options for intervention will allow tailored approaches to be used depending on the endemic location and associated support systems, including local difficulties with distribution. Each country could make appropriate decisions on the treatment approach after full consideration of its own various local situations. To fit this "menu" approach, programs should perhaps designate their endemic areas (foci) as individual projects rather than using a whole-country approach. Obviously, cross-border endemic areas will need to be addressed, and positive interactions between the programs of the different countries are encouraged. 
As described above, there have been considerable changes in the programs to deal with onchocerciasis over the past 40 years, one of which has been the focusing of what are known as the NTDs into a collective target for "eradication, elimination, and control" through a declaration in February 2012 led by WHO. There, a WHO roadmap to accelerate work to overcome these diseases was announced and joined by a wide range of public and private partners that included donors foundations, drug companies, and governmental bodies in an effort termed the London Declaration. This combining of efforts against a range of often coexisting diseases requires new medical and logistic approaches.

Different drugs often need to be combined or at least given in close temporal proximity and this has often required changes to long standing onchocerciasis programs in some countries. Success for the WHO Roadmap is set for 2020 for the elimination of diseases such as onchocerciasis; this is an ambitious goal but it is likely that onchocerciasis will be one of the most successful of the diseases that are targeted by the London Declaration.

\section{The search for new drugs}

As discussed above, there is an ongoing search for new drugs that will safely kill adult filariae (Table 2). This elusive target probably needs to be brought into the field within the next 2-3 years if it is to be a major contribution to the elimination target of 2025. In addition to following the standard approaches to screening for new drugs against an infectious agent, it is also important to understand the mechanism by which new or old drugs damage the various components of adult filariae (Figure 3). This information will most likely facilitate the discovery of agents that do not cause rapid death of the adults, and importantly avoid acute adverse reactions such as abscesses. It is believed that a drug that induces slow degenerative changes in these parasites that are eventually lethal will elicit minimal or no side reactions; this is the case with doxycycline. As many screening programs are based solely on evaluation of motility, and motility does not necessarily reflect the necessary permanent damage to internal organs such as the uterus; it is quite possible that promising hits may be missed in such screens. Finding agents that induce slow degenerative changes would be greatly facilitated by more basic research on the action of drugs that might act this way. An example of another drug that appears to cause these kinds of effects is flubendazole, an agent that damages the worm's uterine tissues, perhaps irreversibly, and causes a slow loss of viability.
It should be noted that ivermectin was used in the field for over 20 years before investigations really began to understand how the drug works. Indeed, concerns about the possible development of ivermectin resistance are better addressed in light of improved knowledge about how this drug works. A current candidate macrofilaricide, flubendazole, has been used extensively in animal models despite the fact that we poorly understood how the drug kills and/or sterilizes adult filariae; it is known to inhibit tubulin polymerization as the mechanism of action, but we do not yet know why this effect is slowly lethal to the parasite. Although the end point of safe effectiveness is the major driving criterion, it is likely that a better understanding of the mechanisms of drug action and measurement of these will greatly enhance drug development.

An area often forgotten in discussions of the role of chemotherapy of parasites is the function of the host immune system subsequent to the administration of a chemotherapeutic agent. The degeneration of parasites after chemotherapy releases large amounts of previously unexposed antigens, and perhaps with untoward consequences, elimination of parasite factors that were keeping the immune system in a clinically quiescent state before treatment. Filarial infections are prime examples of the involvement of the immune system in the host response to the parasite and to the clinical presentations following anthelmintic treatment; the anaphylactoid reactions seen after DEC and other agents that comprise the Mazzotti reaction underscore this. More recently, the immune system has been directly implicated in the action of ivermectin as a microfilaricide. ${ }^{44,46}$ Thus, it is important to keep the immune system in mind when investigating the consequences of drug treatment.

Immune responses to parasites began to be better understood in the 1980 s, catalyzed by the hope that an immunotherapeutic approach (vaccine) for onchocerciasis might be developed. However, immunotherapy for parasitic diseases has proven to be extremely difficult with rare exceptions. A vaccine against onchocerciasis has not been developed, and is not likely to be available before the 2025 goal for elimination. Chemotherapy remains the predominant approach, but must be deployed with an open eye for improvements in drug distribution and post-campaign care of those affected. Prophylaxis of onchocerciasis has never been a major goal of research agencies, partly due to the fact that this is a chronic and relatively slowly acquired infection. However, more pertinently, treatment programs have been directed against populations in which infection is already prevalent. Even if an agent that is effective against the early 


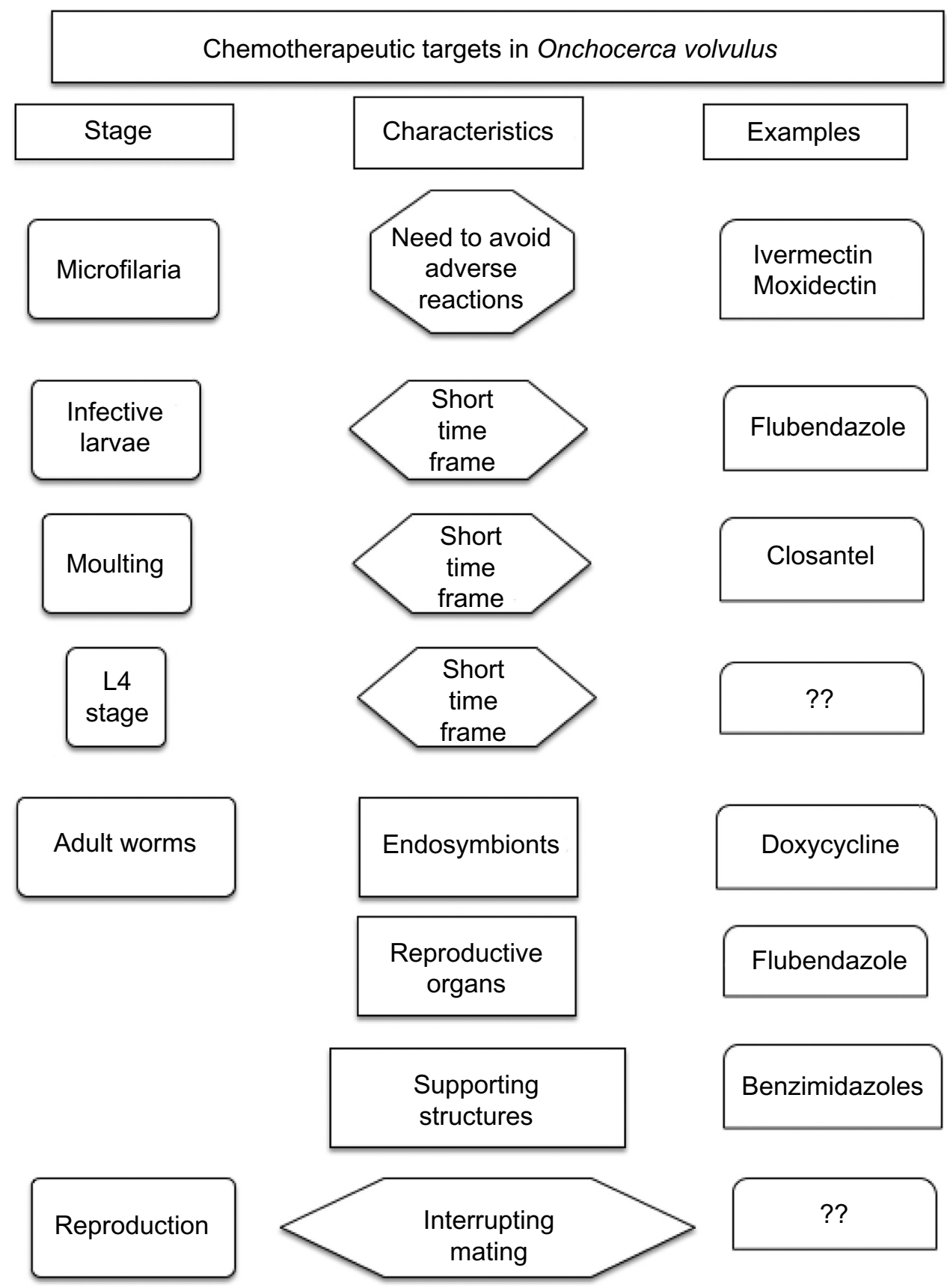

Figure 3 Targets within Onchocerca volvulus and its life cycle that may be susceptible to chemotherapeutic attack. Abbreviations: ??, none defined; L4, fourth stage larvae.

stages of $O$. volvulus infection could be identified, the need for frequent dosing to eliminate newly infecting parasites is unlikely to be compatible with MDA programs that are sustainable in many endemic areas.

An important fact when developing a new drug for treatment of a disease such as onchocerciasis, one that almost exclusively affects people in developing countries, is the likely need for donation by a major manufacturer. When Merck \& Co, Inc. entered into a public-private partnership to donate and distribute ivermectin, a precedent was created that has been followed by several other companies. Merck KG, GlaxoSmithKline plc (London, UK), Johnson \& Johnson (New Brunswick, NJ, USA), and Eisai Inc. (Woodcliff Lake, NJ, USA). have all committed to donate drugs for soil-transmitted nematodes, onchocerciasis, lymphatic filariasis, and schistosomiasis, and any new drug for NTDs may also need a manufacturer who will donate it; there will at least need to be a plan for funding its production and distribution. This needs to be an early consideration in any development program for a new drug for onchocerciasis 
and other NTDs. The integration of antiparasite programs in the last decade ${ }^{65}$ has also catalyzed the need to understand how treatments used for one disease may affect others; overlaps in treatment will have obvious clinical benefits and can provide a way to reduce the distribution burden for country programs (Table 1).

There are reasons to be hopeful about prospects for introducing a new macrofilaricide in time to assist in the elimination campaign. Flubendazole (mentioned above) is currently approved for human use as a very effective anthelmintic for treatment of gastrointestinal nematode infections. It showed considerable promise in earlier studies in the 1970s-1980s as a macrofilaricide against multiple species of filariae when administered parenterally, and is now being reformulated to markedly enhance its oral bioavailability. ${ }^{38}$ Because of its insolubility, flubendazole has been a challenge to drug developers; however, modern pharmaceutical chemistry approaches have led to new formulations that provide excellent oral bioavailability. These new formulations are currently being evaluated for safety and efficacy in preclinical models; it is hoped to go in human trials in 2015.

Moxidectin, a semisynthetic macrocyclic lactone related to ivermectin, is another potentially useful agent for onchocerciasis chemotherapy derived from veterinary medicine. ${ }^{66}$ It has undergone initial trials in humans and has been shown to be a highly efficacious and exceptionally persistent microfilaricide ${ }^{67,68}$ (Figure 2). Recent studies suggest that it is a more effective compound than ivermectin against $O$. volvulus $\mathrm{mf}$, but has no marked effect on adult parasites, and thus is probably not a useful macrofilaricide. ${ }^{66}$ Although moxidectin is not registered for use in humans, it is a candidate that could be considered in a panel of drugs that might be made available for the final push toward elimination of this disease.

Other potential drugs for onchocerciasis have been proposed in recent years, ${ }^{8}$ although none has shown definitive potential for being a major contributor to elimination programs. Closantel, a salicylanilide used in veterinary medicine to treat liver fluke (Fasciola spp.) infections, was suggested as a candidate, ${ }^{69}$ however, this drug appears to only affect molting stages of $O$. volvulus (Figure 2) and is not a candidate macrofilaricide. It is possible that closantel could be used as a prophylactic agent, but as discussed above, prophylaxis is not a major goal for an MDA program. It could be argued that this drug might be useful in maintaining elimination of the infection from a cleared area, if this was ever required, but it would not seem to be a priority for development at this time. Among the other compounds that have been proposed in recent times as being antifilarial is the kinase inhibitor imatinib, which is also active against Schistosoma sp. ${ }^{70}$ and the filarial parasite Brugia malayi. ${ }^{71}$ Imatinib is currently used for the treatment of chronic myelogenous leukemia and thus has been tested for use in humans at least in these cases. It is possible that imatinib may be included in a battery of chemotherapeutic options for the treatment of onchocerciasis, although it has not been tested in this disease and cost-of-goods may be a challenge. Finally, the semisynthetic cyclic depsipeptide emodepside, used in veterinary medicine to control gastrointestinal nematodiases of companion animals, has potent activity against Onchocerca adults in culture ( see $^{10}$ for review), but activity in animal models of filariasis is highly species-dependent. ${ }^{71}$

\section{General discussion}

Onchocerciasis has moved over the last 30 years from a position of clinical notoriety characterized by blindness and disability, flagged by disturbing images of blind people in Africa and elsewhere, to one of success as the ivermectin MDA programs have progressed. Indeed, it currently has a positive image as one of the most important success stories in tropical medicine today. This has come about through a remarkable donation program based on a single drug, ivermectin, a drug that has been a mainstay of veterinary medicine since its discovery in the late 1970 s. The donation of ivermectin for onchocerciasis has changed in major ways access to health for many people living in the most remote parts of Africa and other developing areas. It should be emphasized that the success of the original ivermectin donation program, which began in $1987,{ }^{72}$ has catalyzed similar programs for a number of other parasitic and tropical diseases. This success, however, has meant that government health programs in many developing countries in tropical regions have now a number of diseases that come under MDA programs rather than only river blindness; this in itself has posed financial and management challenges to these countries and this issue should be kept in mind when discussing how to support and interact with country programs.

Ivermectin, now used with albendazole in many locations, has reduced the prevalence and incidence of onchocerciasis in many countries - indeed, some countries, with small endemic areas, are now looking at complete elimination of the transmission in those areas. However, challenges remain in much of Africa's endemic areas. Addressing these challenges centers around optimizing existing MDA programs, using all means available, enhancing the panel of drugs and procedures available by adding an effective macrofilaricide, 
and maintaining the global efforts to focus on the goal of elimination by 2025 .

Although there have been remarkable successes with ivermectin for treating onchocerciasis, such as the elimination of infection from countries in Latin America and isolated foci in Africa, it is likely that a new safe and effective macrofilaricide, or at least a way of permanently suppressing microfilarial production, will be needed to achieve complete elimination of this infection in Africa. Candidate macrofilaricides have recently emerged along with reconsideration of the global management strategies necessary to distribute such an agent. It is hoped that the goal for global eradication of this infection by 2025 will be achieved using such agents if the current focus and support of this goal is maintained.

Scientific resources, including those of the pharmaceutical world and academia, together with endemic country personnel to focus on this important goal. Success in macrofilaricide discovery and development will bring greater understanding of the biology of this parasite and the pharmacology of existing therapeutic agents. Success in global eradication of onchocerciasis will be a major global medical event, and will be to the credit of partnerships between academic scientists, pharmaceutical companies, philanthropists, and, most importantly, the medical communities that deliver health care in the endemic countries.

\section{Disclosure}

The authors report no conflicts of interest in this work.

\section{References}

1. Centers for Disease Control and Prevention (CDC). Progress toward elimination of onchocerciasis in the Americas - 1993-2012. MMWR Morb Mortal Wkly Rep. 2013;62:405-408.

2. Burki T. River blindness elimination in Columbia. Lancet Inf Dis. 2013;13:922-923.

3. Lovato R, Guevara A, Guderian R, et al. Interruption of infection transmission in the onchocerciasis focus of Ecuador leading to the cessation of ivermectin distribution. PLoS Negl Trop Dis. 2014;8:e2821.

4. Higazi TB, Zarroug IM, Mohamed HA, et al. Interruption of Onchocerca volvulus transmission in the Abu Hamed focus, Sudan. Am J Trop Med Hyg. 2013;89:51-57.

5. Rodríguez-Pérez MA, Lutzow-Steiner MA, Segura-Cabrera A, et al. Rapid suppression of Onchocerca volvulus transmission in two communities of the Southern Chiapas focus, Mexico, achieved by quarterly treatments with Mectizan. Am J Trop Med Hyg. 2008;79: 239-244.

6. Rodríguez-Pérez Mario A, Domínguez-Vázquez A, Unnasch TR, et al. Interruption of transmission of Onchocerca volvulus in the Southern Chiapas focus, Mexico. PLoS Negl Trop Dis. 2013;7:e2133.

7. Mackenzie CD, Homeida MM, Hopkins AD, Lawrence JC. Elimination of onchocerciasis from Africa: possible? Trends Parasitol. 2012;28: $16-22$.

8. Mackenzie CD, Geary TG. Addressing the current challenges to finding new anthelminthic drugs. Expert Rev Anti Infect Ther. 2013;11: $539-541$.
9. Singh PK, Ajay A, Kushwaha S, Tripathi RP, Misra-Bhattacharya S Towards novel antifilarial drugs: challenges and recent developments. Future Med Chem. 2010;2:251-283.

10. Geary TG, Mackenzie CD. Progress and challenges in the discovery of macrofilaricidal drugs. Expert Rev Anti Infect Ther. 2011;9:681-695.

11. Anderson J, Fuglsang H. Further studies on the treatment of ocular onchocerciasis with diethylcarbamazine and suramin. Br JOphthalmol. 1978;62:450-457.

12. Orange RP, Austen KF. Prospects in asthma therapy: disodium cromoglycate and diethylcarbamazine. $N$ Engl J Med. 1968;279: $1055-1057$.

13. Gardon J, Gardon-Wendel N, Demanga-Ngangue, Kamgno J, Chippaux JP, Boussinesq M. Serious reactions after mass treatment of onchocerciasis with ivermectin in an area endemic for Loa loa infection. Lancet. 1997;350:18-22.

14. Kamgno J, Boussinesq M, Labrousse F, Nkegoum B, Thylefors BI, Mackenzie CD. Encephalopathy after ivermectin treatment in a patient infected with Loa loa and Plasmodium spp. Am J Trop Med Hyg. 2008;78:546-551.

15. Brieger WR, Okeibunor JC, Abiose AO, et al. Compliance with eight years of annual ivermectin treatment of onchocerciasis in Cameroon and Nigeria. Parasit Vectors. 2011;4:152.

16. Gustavsen K, Hopkins A, Sauerbrey M. Onchocerciasis in the Americas: from arrival to (near) elimination. Parasit Vectors. 2011;4:205

17. Molyneux D. Lymphatic Filariasis (Elephantiasis) elimination: A public health success and development opportunity. Filaria J. 2003;2:13.

18. Anderson J, Fuglsang H, de C Marshall TF. Effects of suramin on ocular onchocerciasis. Tropenmed Parasitol. 1976;27:279-296.

19. Fernández de Castro J. Historia de la Oncocercosis. Salud Púb México. 1979;21:683-696. [Spanish].

20. Guderian RH. Effects of nodulectomy in onchocerciasis in Ecuador. Trop Med Parasitol. 1988;39 Suppl 4:356-357.

21. Mazzotti L. Observations on the use of hetrazan in onchocerciasis in Mexico. Am J Trop Med Hyg. 1951;31:628-632.

22. Francis H, Awadzi K, Ottesen EA. The Mazzotti reaction following treatment of onchocerciasis with diethylcarbamazine: clinical severity as a function of infection intensity. Am J Trop Med Hyg. 1985;34: $529-536$.

23. Awadzi K, Opoku NO, Addy ET, Quartey BT. The chemotherapy of onchocerciasis. XIX: The clinical and laboratory tolerance of high dose ivermectin. Trop Med Parasitol. 1995;46:131-137.

24. Janssens PG. [Therapeutic trials of Mel W in onchocerciasis and loaiasis]. Ann Soc Belg Med Trop (1920). 1961;41:373-380. French.

25. Friedheim EA. Mel W in the treatment of onchocerciasis in leprosy patients: results in 12 cases observed for 20 months after treatment. Ann Trop Med Parasitol. 1962;56:343-351.

26. Ghalal I. The safety and efficacy of the Sudan (Sherif Dawood) Regimen of suramin therapy in the treatment of onchocerciasis. Sudan Med J. 1985;21(Suppl):89-94.

27. Cross HF, Bronsvoort BM, Wahl G, Renz A, Achu-Kwi D, Trees AJ. The entry of ivermectin and suramin into Onchocerca ochengi nodules. Ann Trop Med Parasitol. 1997;91:393-401.

28. Budden FH. The natural history of ocular onchocerciasis over a period of 14-15 years and the effect on this of a single course of suramin therapy. Trans R Soc Trop Med Hyg. 1976;70:484-491.

29. Bird AC, El-Sheikh H, Anderson J, Fuglsang H. Visual loss during oral diethylcarbamazine treatment for onchocerciasis. Lancet. 1979;2:46.

30. Langham ME, Traub ZD, Richardson R. A transepidermal chemotherapy of onchocerciasis. Tropenmed Parasitol. 1978;29:156-162.

31. Mackenzie CD, Kron MA. Diethylcarbamazine: a review of its action in onchocerciasis, lymphatic filariasis and inflammation. Trop Dis Bull. 1985;83:R1-R34.

32. Stingl P, Ross M, Gibson DW, Ribas J, Connor DH. A diagnostic "patch test" for onchocerciasis using topical diethylcarbamazine. Trans $R$ Soc Trop Med Hyg. 1984;78:254-258. 
33. Guderian RH, Proano JR, Mackenzie CD, Herdoiza M. La aplicacion de una preuba dermoreactiva, simple y segura para la oncocercosis. [Application of a simple and safe skin test for onchocerciasis]. Rev Facultad Ciencias (Quito, Ecuador). 1986;11:9-13. [Spanish].

34. Toè L, Adjami AG, Boatin BA, et al. Topical application of diethylcarbamazine to detect onchocerciasis recrudescence in west Africa. Trans R Soc Trop Med Hyg. 2000;94:519-525.

35. Rivas-Alcala R, Mackenzie CD, Gomez-Rojo E, Greene BM, Taylor HR. The effects of diethylcarbamazine, mebendazole and levamisole on Onchocerca volvulus in vivo and in vitro. Tropenmed Parasitol. 1984;35:71-77.

36. Dominguez-Vazquez A, Taylor HR, Green BM, et al. Comparison of flubendazole and diethylcarbamazine in treatment of onchocerciasis. Lancet. 1983;1:139-143.

37. Cline BL, Hernandez JL, Mather FJ, et al. Albendazole in the treatment of onchocerciasis: double-blind clinical trial in Venezuela. Am J Trop Med Hyg. 1992;47:512-520.

38. Mackenzie CD, Geary TG. Flubendazole: a candidate macrofilaricide for lymphatic filariasis and onchocerciasis field programs. Expert Rev Anti Infect Ther. 2011;9:497-501.

39. Poltera AA, Zea-Flores G, Guderian R, et al. Onchocercacidal effects of amocarzine (CGP 6140) in Latin America. Lancet. 1991;337(8741): $583-584$.

40. Mahmoud BM, Vandewaa EA, Geary TG, Guderian R, Williams JF. Uptake of chloroquine by Onchocerca volvulus in vivo and in vitro. Ann Trop Med Parasitol. 1991;85:523-528.

41. Kale OO. Further trials of mebendazole and metrifonate in the treatment of onchocerciasis. Bull World Health Organ. 1982;60:109-113.

42. Geary TG. Are new anthelmintics needed to eliminate human helminthiases? Curr Opin Infect Dis. 2012;25:709-717.

43. Geary TG, Moreno Y. Macrocyclic lactone anthelmintics: spectrum of activity and mechanism of action. Curr Pharm Biotechnol. 2012;13: 866-872.

44. Moreno Y, Nabhan JF, Solomon J, Mackenzie CD, Geary TG. Ivermectin disrupts the function of the excretory-secretory apparatus in microfilariae of Brugia malayi. Proc Natl Acad Sci U SA. 2010;107: 20120-20125.

45. Nana-Djeunga HC, Bourguinat C, Pion SD, et al. Reproductive status of Onchocerca volvulus after ivermectin treatment in an ivermectin- naïve and a frequently treated population from Cameroon. PLoS Negl Trop Dis. 2014;8:e2824.

46. Ali MM, Baraka OZ, AbdelRahman SI, et al. Immune responses directed against microfilariae correlate with severity of clinical onchodermatitis and treatment history. $J$ Infect Dis. 2003;187:714-717.

47. Kozek WJ, Rao RU. The discovery of wolbachia in arthropods and nematodes - a historical perspective. Issues Infect Dis. 2007;5: $1-14$.

48. Hoerauf A, Mand S, Adjei O, Fleischer B, Büttner DW. Depletion of Wolbachia endobacteria in Onchocerca volvulus by doxycycline and microfilaridermia after ivermectin treatment. Lancet. 2001;357(9266): $1415-1416$

49. Hoerauf A, Mand S, Volkmann L, et al. Doxycycline in the treatment of human onchocerciasis: Kinetics of Wolbachia endobacteria reduction and inhibition of embryogenesis in female Onchocerca worms. Microbes Infect. 2003;5:261-273.

50. Hoerauf A, Specht S, Marfo-Debrekyei Y, et al. Efficacy of 5-week doxycycline treatment on adult Onchocerca volvulus. Parasitol Res. 2009; 104:437-447.

51. Gardon J, Boussinesq M, Kamgno J, Gardon-Wendel N, Demanga-Ngangue, Duke BO. Effects of standard and high doses of ivermectin on adult worms of Onchocerca volvulus: a randomised controlled trial. Lancet. 2002;360:203210.

52. Pacqué M, Muñoz B, Poetscke G, Foose J, Greene BM, Taylor HR. Pregnancy outcome after inadvertent ivermectin treatment during community-based distribution. Lancet. 1990;336:1486-1489.
53. Gyapong JO, Chinbuah MA, Gyapong M. Inadvertent exposure of pregnant women to ivermectin and albendazole during mass drug administration for lymphatic filariasis. Trop Med Int Health. 2003;8:1093-1101.

54. Chippaux JP, Gardon-Wendel N, Gardon J, Ernould JC. Absence of any adverse effect of inadvertent ivermectin treatment during pregnancy. Trans R Soc Trop Med Hyg. 1993;87:318.

55. Tamarozzi F, Tendongfor N, Enyong PA, et al. Long term impact of large scale community-directed delivery of doxycycline for the treatment of onchocerciasis. Parasit Vectors. 2012;5:53.

56. Mackenzie CD, Geary TG, Gerlach JA. Possible pathogenic pathways in the adverse clinical events seen following ivermectin administration to onchocerciasis patients. Filaria J. 2003;2 Suppl 1:S5.

57. Richards FO Jr, Eberhard ML, Bryan RT, et al. Comparison of high dose ivermectin and diethylcarbamazine for activity against bancroftian filariasis in Haiti. Am J Trop Med Hyg. 1991;44:3-10.

58. Geary TG, Bourguinat C, Prichard RK. Evidence for macrocyclic lactone anthelmintic resistance in Dirofilaria immitis. Top Companion Anim Med. 2011;26:186-192.

59. Osei-Atweneboana MY, Eng JK, Boakye DA, Gyapong JO, Prichard RK. Prevalence and intensity of Onchocerca volvulus infection and efficacy of ivermectin in endemic communities in Ghana: a two-phase epidemiological study. Lancet. 2007;369:2021-2029.

60. Nana-Djeunga H, Bourguinat C, Pion SD, et al. Single nucleotide polymorphisms in $\beta$-tubulin selected in Onchocerca volvulus following repeated ivermectin treatment: possible indication of resistance selection. Mol Biochem Parasitol. 2012;185:10-18.

61. Connor DH, Gibson DW, Neafie RC, Merighi B, Buck AA. Sowda onchocerciasis in north Yemen: a clinicopathologic study of 18 patients. Am J Trop Med Hyg. 1983;32:123-137.

62. Ghalib HW, Mackenzie CD, Kron MA, Williams JF, el Khalifa M, el Sheikh H. Severe onchocercal dermatitis in the Ethiopian border region of Sudan. Ann Trop Med Parasitol. 1987;81:405-419.

63. Baraka OZ, Mahmoud BM, Ali MM, et al. Ivermectin treatment in severe asymmetric reactive onchodermatitis (sowda) in Sudan. Trans R Soc Trop Med Hyg. 1995;89:312-315.

64. Traore MO, Sarr MD, Badji A, et al. Proof-of-principle of onchocerciasis elimination with ivermectin treatment in endemic foci in Africa: final results of a study in Mali and Senegal. PLoS Negl Trop Dis. 2012;6:e1825.

65. Johnston KL, Ford L, Taylor MJ. Overcoming the challenges of drug discovery for neglected tropical diseases: The A.WOL experience. J Biomol Screen. 2014;19:335-343.

66. Cotreau MM, Warren S, Ryan JL, et al. The antiparasitic moxidectin: safety, tolerability, and pharmacokinetics in humans. J Clin Pharmacol. 2003;43:1108-1115.

67. World Health Organization. African Programme for Onchocerciasis Control (APOC): Final Communiqué. 2012. In 17th Session of the Joint Action Forum, Kuwait City, Kuwait, 12-14 December, 2011, WHO. Available from: http://www.who.int/apoc/about/structure/jaf/JAF17_Final_ Communique_030112Clean.pdf?ua=1. Accessed October 10, 2014.

68. Awadzi K, Opoku NO, Attah SK, Lazdins-Helds J, Kuesel AC. A randomized, single-ascending-dose, ivermectin-controlled, double-blind study of moxidectin in Onchocerca volvulus infection. PLoS Negl Trop Dis. 2014;8:e2953.

69. Gloeckner C, Garner AL, Mersha F, et al. Repositioning of an existing drug for the neglected tropical disease Onchocerciasis. Proc Natl Acad Sci U S A. 2010;107:3424-3429.

70. Beckmann S, Grevelding CG. Imatinib has a fatal impact on morphology, pairing stability and survival of adult Schistosoma mansoni in vitro. Int J Parasitol. 2010;40:521-526.

71. Zahner H, Taubert A, Harder A, von Samson-Himmelstjerna G. Effects of Bay 44-4400, a new cyclodepsipeptide, on developing stages of filariae (Acanthocheilonema viteae, Brugia malayi, Litomosoides sigmodontis) in the rodent Mastomys coucha. Acta Trop. 2001;80:19-28.

72. Hopkins AD. Ivermectin and onchocerciasis: is it all solved? Eye (Lond). 2005;19:1057-1066. 
Research and Reports in Tropical Medicine

Dovepress

\section{Publish your work in this journal}

Research and Reports in Tropical Medicine is an international, peerreviewed, open access journal publishing original research, case reports, editorials, reviews and commentaries on all areas of tropical medicine, including: Diseases and medicine in tropical regions; Entomology; Epidemiology; Health economics issues; Infectious disease; Laboratory

science and new technology in tropical medicine; Parasitology; Public health medicine/health care policy in tropical regions; and Microbiology. The manuscript management system is completely online and includes a very quick and fair peer-review system. Visit http://www.dovepress. com/testimonials.php to read real quotes from published authors.

Submit your manuscript here: http://www.dovepress.com/research-and-reports-in-tropical-medicine-journal 